\title{
Whole lung lavage therapy for pulmonary alveolar proteinosis: a global survey of current practices and procedures
}

Ilaria Campo ${ }^{1,12^{*+}}$ D , Maurizio Luisetti ${ }^{1+}$, Matthias Griese ${ }^{2}$, Bruce C. Trapne $I^{3}$, Francesco Bonella ${ }^{4}$, Jan Grutters ${ }^{5}$, Koh Nakata ${ }^{6}$, Coline H. M. Van Moorsel ${ }^{5}$, Ulrich Costabel ${ }^{4}$, Vincent Cottin 7 , Toshio Ichiwata ${ }^{8}$, Yoshikazu Inoue $^{9}$, Antonio Braschi ${ }^{10}$, Giacomo Bonizzoni ${ }^{1}$, Giorgio A. Iotti ${ }^{10}$, Carmine Tinelli ${ }^{11}$, Giuseppe Rodi ${ }^{10}$ and for the WLL International Study Group

\begin{abstract}
Background: Whole lung lavage (WLL) is the current standard of care treatment for patients affected by pulmonary alveolar proteinosis (PAP). However, WLL is not standardized and international consensus documents are lacking. Our aim was to obtain a factual portrayal of WLL as currently practiced with respect to the procedure, indications for its use, evaluation of therapeutic benefit and complication rate.
\end{abstract}

Methods: A clinical practice survey was conducted globally by means of a questionnaire and included 27 centers performing WLL in pediatric and/or adult PAP patients.

Results: We collected completed questionnaires from 20 centres in 14 countries, practicing WLL in adults and 10 centers in 6 countries, practicing WLL in pediatric patients.

WLL is almost universally performed under general anesthesia, with a double-lumen endobronchial tube in two consecutive sessions, with an interval of 1-2 weeks between sessions in approximately $50 \%$ of centres. The use of saline warmed to $37^{\circ} \mathrm{C}$, drainage of lung lavage fluid by gravity and indications for WLL therapy in PAP were homogenous across centres.

There was great variation in the choice of the first lung to be lavaged: $50 \%$ of centres based the choice on imaging, whereas $50 \%$ always started with the left lung. The choice of position was also widely discordant; the supine position was chosen by $50 \%$ of centres. Other aspects varied significantly among centres including contraindications, methods and timing of follow up, use of chest percussion, timing of extubation following WLL and lung isolation and lavage methods for small children. The amount of fluid used to perform the WLL is a critical aspect. Whilst a general consensus exists on the single aliquot of fluid for lavage (around $800 \mathrm{ml}$ of warm saline, in adults) great variability exists in the total volume instilled per lung, ranging from 5 to 40 liters, with an average of 15.4 liters/lung.

Conclusions: This international survey found that WLL is safe and effective as therapy for PAP. However these results also indicate that standardization of the procedure is required; the present survey represents the a first step toward building such a document.

Keywords: Pulmonary alveolar proteinosis, Whole lung lavage, Rare disease, Interstitial lung disease

\footnotetext{
* Correspondence: i.campo@smatteo.pv.ti

${ }^{\dagger}$ Equal contributors

'Pneumology Unit, Fondazione IRCCS Policlinico San Matteo and University

of Pavia, Pavia, Italy

${ }^{12}$ Laboratorio di Biochimica e Genetica, S.C. Pneumologia, Fondazione IRCCS

Policlinico San Matteo, via Taramelli 5, 27100 Pavia, Italy

Full list of author information is available at the end of the article
} 


\section{Background}

Whole lung lavage (WLL) is a therapeutic procedure [1] used to treat pulmonary alveolar proteinosis (PAP), a rare syndrome occurring in a heterogeneous group of lung diseases characterized by accumulation of lipoproteinaceous material in the alveoli, whicht impairs oxygen uptake and causes hypoxemic respiratory failure [2, 3]. Therapeutic efficacy derives from removal of the accumulated lipoproteinaceous material - primarily surfactant and necrotic cell debris - by physically 'washing' the alveoli with saline. WLL is usually performed under general anesthesia with lung separation obtained by a double-lumen endobronchial tube. While mechanical ventilation is maintained in one lung, the contralateral lung is repeatedly filled with saline and then drained by gravity. Typically, the lavage is accompanied by chest percussion to emulsify the surfactant sediment, and is continued until the lavage fluid becomes clear, usually judged by visual inspection.

Presently applied WLL procedures are based on the first description by Juan Ramirez Rivera in 1963 [1], but several centres have introduced modifications with the aim of improving the original method [4]. Although widely considered as the standard-of-care for autoimmune PAP [5], the WLL procedure, indications for its use, and the criteria to measure outcome have not been standardized among centres. Nor has the therapeutic effectiveness been compared for different secondary PAP syndromes. Further, centre-specific indications have never been compared, integrated to optimize or standardize WLL, or systematically disseminated. Moreover training on how to perform WLL involves an apprenticeship or in many cases is self taught; which obviously leads to additional variations among centres.

To begin laying the foundation for a consensus and experience-based, best-practice standardization of WLL, physicians performing WLL were surveyed by questionnaire on a global scale. Results concern the practice of WLL in adult and paediatric patients and include information on the procedure itself, local modifications, indications for use, contraindications, procedural monitoring, criteria to measure outcomes and complication rates.

\section{Methods}

\section{WLL questionnaire development}

This study was conducted as part of the e-RARE 2009 EuPAPnet study (http://www.alveolarproteinosis.eu/). An international ad hoc committee of physicians performing WLL and/or caring for PAP patients developed the survey questionnaire, which includes sections for physicians only performing WLL in either adult ( $\geq 18$ years-old) or paediatric (<18 years-old) patients; only lobar or segmental bronchoscopic lavage (LSBL) in adult and/or paediatric patients; or both WLL and LSBL. The questionnaire is available in the Additional file 1.

\section{Data collection}

The study cohort included physicians performing therapeutic lung lavage. Potential partecipants were identified either through a PubMed search (http:// www.ncbi.nlm.nih.gov/pubmed) using the search terms 'pulmonary proteinosis', 'alveolar proteinosis', 'alveolar phospholipidosis,' 'lung lavage', and 'whole lung lavage'; or invited by an announcement placed in the European Respiratory Journal [6] or directly contacted: i.e., members of the international paediatric interstitial lung disease network; and acquaintances of steering committee members. Potential respondents were contacted by e-mail, interested individuals received the questionnaire by email, and all completed questionnaires that were returned were included in the analysis. All respondents gave written authorization for their responses to be included in the study.

\section{Analysis}

Numeric variables were evaluated for normality with the Shapiro-Wilk test and expressed as the mean +/- SD or median $+/$ - the interquartile range (IQR) as appropriate. Comparisons were made by one-way ANOVA with the Bonferroni correction. Categorical data were summarized numerically or expressed as a percentage and compared using the chi square or Fisher's exact test as appropriate. Correlations among continuous variables were made using Pearson's correlation coefficient. All comparisons were two-sided. $\mathrm{P}$ values of $<0.05$ were considered statistically significant. Analyses were performed using STATA, version 13.1 (Stata Corporation, College Station, Texas, USA). Three categories for the number of times WLL was conducted in a single patient (WLL $<2, \geq 2$ WLL $<3$, WLL $>3$ ) were utilized in analyzing results related to clinical outcomes.

Data were considered in a two-stage IPD metaanalysis, in order to correct the unavailability of single patient data. In the first stage, each individual study was analyzed as described in the meta-analysis protocol or analysis plan. As a second step, the results, or summary statistics, of each of these individual study analyses were combined to provide a pooled estimate of effect, as for a conventional systematic review.

\section{Results}

Of the 79 centres identified worldwide that provide therapeutic lung lavage (52 centres in adults, 27 in children), 40 (50\%) expressed an interest in the study and were sent the questionnaire; of these 27 (33\%) completed and returned the documents (Table 1). Among these, seventeen centres treat only adults (14 only WLL, 
Table 1 Centres participating in the survey

\begin{tabular}{|c|c|c|c|c|}
\hline Centre & WLL & SLBL & Adult & Paediatric \\
\hline Sheffield Children's Hospital, UK & $\mathrm{x}$ & $x$ & & $x$ \\
\hline Royal Brompton Hospital, London, UK & $x$ & $x$ & $x$ & $x$ \\
\hline Sheba Medical Centre, Tel-Hashomer, Tel Aviv U., Israel & $x$ & & $x$ & $x$ \\
\hline Children's Hospital Boston, USA & $x$ & & & $x$ \\
\hline Kinderklinik und Kinderpoliklinik im, U. of Munich, Germany & $x$ & $x$ & & $x$ \\
\hline Cincinnati Children's Hospital, USA & $x$ & $x$ & & $x$ \\
\hline Fondazione IRCCS Policlinico San Matteo, Pavia, Italy & x & & $x$ & \\
\hline University of North Carolina at Chapel Hill, USA & & $x$ & & $x$ \\
\hline Hacettepe University, Ankara, Turkey & & $x$ & & $x$ \\
\hline U. Witwatersrand, Johannesburg, South Africa & & $x$ & & $x$ \\
\hline Pavlov State Medical University, St. Petersburg, Russian Federation & X & $x$ & $x$ & \\
\hline NHO Kinki-Chuo Chest Medical Centre, Osaka, Japan & X & $x$ & $x$ & \\
\hline U. Medical College, Hangzhou, Zhejiang, China & X & $x$ & $x$ & \\
\hline Lungenclinic Grosshandorf, Germany & $x$ & & $x$ & \\
\hline U. Hospital, Olomouc, Czech Republic & X & & $x$ & \\
\hline Asklepios-Fachkliniken München Gauting, Germany & $x$ & & $x$ & \\
\hline Helsinki University Central Hospital, Finland & $x$ & & $x$ & \\
\hline Dept. of Respiratory Medicine, Cork University Hospital, Ireland & $x$ & & $x$ & \\
\hline Care Medicine Thoraxklinik, Heidelberg, Germany & $x$ & & $x$ & \\
\hline Serviço de Pneumologia- Hospital São João-Porto, Portugal & $x$ & & $x$ & \\
\hline Ruhrlandklinik-University of Duisburg Essen, Germany & $x$ & & $x$ & \\
\hline Pulmonary Division, University Hospital, Zurich, Switzerland & $x$ & & $x$ & \\
\hline Hopital Louis Pradel, Lyon, France & $x$ & & $x$ & \\
\hline Tokyo Medical U. Hachioji Medical Centre, Japan & $x$ & & $x$ & \\
\hline Kantonsspidal Aarau, Switzerland & $x$ & & $x$ & \\
\hline St. Antonius Hospital Nieuwegein, The Netherlands & $x$ & & $x$ & \\
\hline Kempten-Oberallgäu Hospital, Immenstadt, Germany & $x$ & & $x$ & $x$ \\
\hline
\end{tabular}

no one only SLBL, 3 both WLL and SLBL), seven treat only children ( 1 only WLL, 3 only SLBL, 3 both WLL and SLBL), and three treat both (2 only WLL, no one only SLBL, 1 both WLL and SLBL). Among centres providing both WLL and SLBL, WLL is considered the standard-of-care and SLBL treatment is reserved for patients judged unable to tolerate WLL. SLBL was also used in young children for whom double lumen endobronchial tubes of sufficiently small diameter are unavailable, and also to predict the therapeutic effectiveness of WLL in patients in whom this was uncertain, e.g., those with Niemann Pick disease.

\section{WLL in Adult Patients}

Twenty centres reported performing WLL in adults (Table 1). The mean $(+/-\mathrm{SD})$ duration of experience was $18+/-11$ years and varied substantially among centres (Fig. 1a). The mean number of WLL procedures performed annually per centre was $5.61+/-5.04$ and also varied among centres (Fig. 1b).

\section{Indications and contraindications for WLL therapy}

Indications for WLL varied among centres (Table 2). Specific indications included an unspecified decline in lung function, a decline in resting $\mathrm{PaO}_{2}$, worsening of lung disease severity judged radiographically based on a comparison of serial chest computed tomograms or chest radiograms (using visual assessment in all except two centres that use semi-quantitative criteria), decline in diffusing carbon monoxide capacity (DLCO), decline in forced vital capacity (FVC), decline in resting oxygen saturation by pulse oximetry $\left(\mathrm{SpO}_{2}\right)$ or an increase in respiratory symptoms.

Three centres also reported using WLL for indications other than PAP, including: accidental inhalation of 
a)

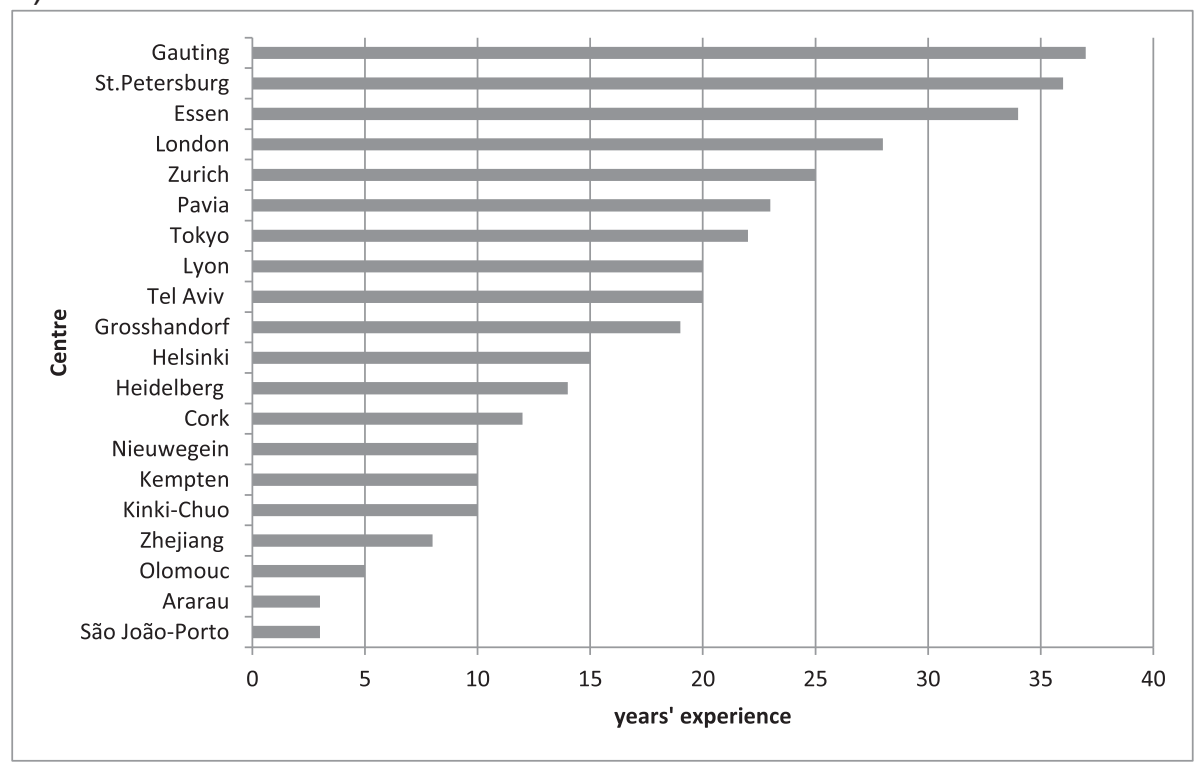

b)

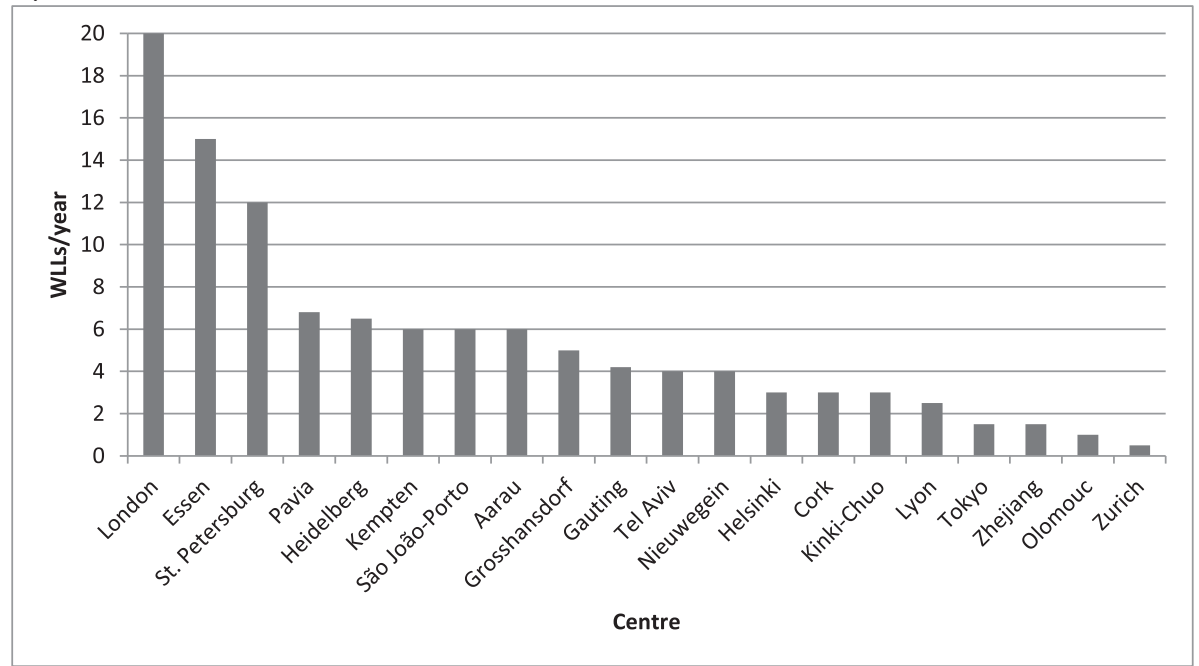

Fig. 1 a. Years' experience of each centre in performing WLL in adult PAP patients. b. Mean number of WLLs performed per centre annually, in adult PAP patients

Table 2 Indications for WLL therapy

\begin{tabular}{ll}
\hline Indications for WLL & \% of centres \\
\hline Unspecified decline in lung function & 100 \\
Decline in resting $\mathrm{PaO}_{2}$ & 90 \\
Chest X-ray or CT & 79 \\
Decline in DLCO & 70 \\
Decline in FVC & 63 \\
Decline in SpO2 & 58 \\
Symptoms & 42 \\
Other & 15 \\
\hline
\end{tabular}

activated charcoal, alveolar hemorrhage, silicoproteinosis, lipoid pneumonia, silicosis or cryptogenic fibrosing alveolitis (CFA).

Contraindications to WLL were applied by six centres and included severe cardiovascular disease, heart failure, sepsis, significant lung infection and end-stage pulmonary fibrosis.

\section{Interval between treatment of right and left lungs}

Most (17/20) centres performed WLL in separate sessions on each lung for a given patient. The time separating WLL therapy for each lung was $2.9 \pm 1.18$ weeks and 


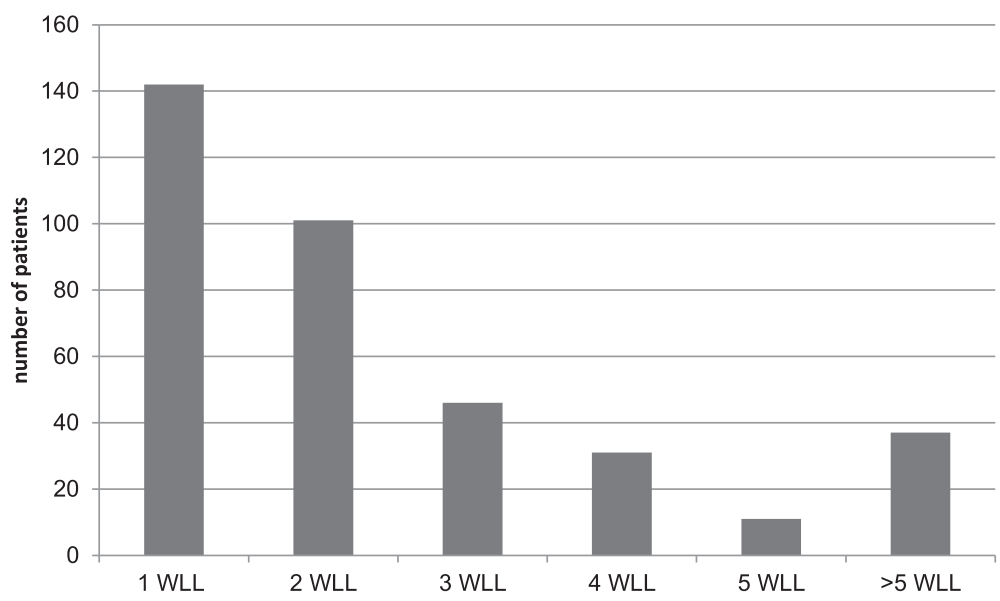

Fig. 2 Stratification of adult autoimmune PAP patients according to the number of WLL procedures received

varied from $24 \mathrm{~h}$ at one centre to 6-12 weeks at another centre. Only one centre routinely treated both lungs in one session, while two other centres treated lungs in one or two sequential sessions depending on patient tolerance.

\section{Number of WLL treatments Per patient required in autoimmune PAP}

Of the approximate 368 PAP patients for whom data was evaluated, the number of WLL procedures received by PAP patients during the follow-up period was $2.5+/-1.5$ in five years. Of these patients, two thirds required one lavage, and only $10 \%$ required more than five WLL procedures (Fig. 2). The interval between consecutive bilateral WLL procedures was $8+/-6.45$ months but varied from 1 week to several years.

Because the clinical course of autoimmune PAP is variable, we evaluated whether the experience of the centre (number of years in activity and number of WLL procedures performed annually) and the volumes used for WLL might relate to clinical outcome. We found no correlation in these parameters $\left(\chi^{2}=0.1238\right.$ and 0.4905 , respectively) suggesting that neither the experience level or volume of WLL fluid used were significantly correlated with clinical outcome.

\section{Complications in WLL}

Based on an estimated 1110 WLL procedures, the centres reported specific complication rates ranging from a median of $16 \%$ for transient fever (the most common complication) to $0.8 \%$ for pneumothorax (Table 3).

\section{Follow up after lung lavage therapy}

All centres reported performing a short-term assessment (chest $\mathrm{x}$-ray and functional assessment) after WLL over a period that ranged from $2 \mathrm{~h}$ to 2 weeks. Some $(9 / 20)$ centres also performed a follow up chest computed tomogram $(\mathrm{CT})$ scan at a variable time ranging from 7 days to 6 months. All centres reported performing medium- to long-term follow up using conventional radiological and functional parameters including chest CT scan $(12 / 20$ centres - routinely at 8 , and on an 'as indicated basis' at 4 months). Six centres performed follow up biomarker evaluations including serum LDH, GM-CSF autoantibody levels and less commonly SPA, SPD, KL-6, CEA, Cyfra 21-1. Two

Table 3 Complications in WLL

\begin{tabular}{ll}
\hline Complication & Frequency $(\%)^{\mathrm{a}, \mathrm{b}}$ \\
\hline Fever & 18.0 \\
Fluid leakage & 4.0 \\
Hypoxemia & 14.2 \\
Wheezing & 6.1 \\
Pneumonia & 5.0 \\
Headache & 0 \\
Respiratory acidosis & 0 \\
Transient neuropathy & 0 \\
Pleural effusion & 3.1 \\
Prolonged mechanical ventilation & 0 \\
Metabolic acidosis & 0 \\
Pulmonary thromboembolism & 0 \\
Pneumothorax & 0.8 \\
Transient cardiac ischemia & 0 \\
Cardiac arrest & $1.1^{c}$
\end{tabular}

${ }^{\mathrm{a}}$ Based on an estimated total of 1110 WLLs. Estimation of the total number of WLLs is the sum of the total per centre calculated as: median WLL number per year multiplied by the number of years' experience

${ }^{b}$ The frequency of complications was estimated as the mean value of frequencies reported by the centres

CCardiac arrest occurred only in 1 case out of 5 WLL reported, only at 1 centre 
centres followed up WLL procedures by using a quality of life questionnaire.

\section{Implementation of the WLL procedure Lung isolation}

The lungs must be isolated during WLL so that one can be lavaged while the other is ventilated to maintain the necessary gas exchange. This is usually achieved by positioning a double-lumen endobronchial tube, which ensure an adequate seal to prevent fluid spillage from the treated lung into the ventilated lung. A left-sided double-lumen endobronchial tube is the most common choice in adults and larger children. The minimum size of the double-lumen tube is $26 \mathrm{Fr}$.

\section{Anesthesia}

At all centres, WLL is performed under general anesthesia (Additional file 2: Table S1), typically by intravenous anesthesia, most commonly with a combination of propofol, an opioid and a neuro-muscular blocking agent. Two centres use volatile anesthetic agents. At about half the centres $(9 / 20)$, a procedure referred to as 'lung atelectasis/degassing' is performed prior to WLL. Patient parameters typically monitored during WLL are indicated in Table 4.

\section{Lung selection}

The method used to decide which lung to treat first varied among centres. About half $(12 / 20)$ used radiographic information to identify and treat the more severely affected lung first, which was based on visual inspection of the chest CT (9 centres), chest x-ray ( 2 centres), or a combination of chest $\mathrm{x}$-ray and CT (1 centre). At six centres, the decision was independent of lung-specific severity: the left lung was always treated first, due to its smaller relative size. At about half of the centres $(9 / 20)$, a procedure referred to as 'lung atelectasis/degassing' is performed prior to starting the lavage of each lung. Degassing of one lung is obtained by ventilation with $100 \%$

Table 4 Parameters monitored during anesthesia/WLL

\begin{tabular}{ll}
\hline Parameter monitored & $\mathrm{N}^{\circ}$ of centres (\%) \\
\hline EKG & $20(100 \%)$ \\
$\mathrm{SaO}_{2}$ & $18(90 \%)$ \\
Non invasive blood pressure & $13(65 \%)$ \\
End tital $\mathrm{CO}_{2}$ & $13(65 \%)$ \\
Arterial catheter blood pressure & $11(55 \%)$ \\
$\mathrm{CVC}$ & $7(35 \%)$ \\
Bispectral index & $3(15 \%)$ \\
Blood gas analysis & $3(15 \%)$ \\
Pulmonary compliance & $1(5 \%)$ \\
\hline
\end{tabular}

oxygen followed by forced lung deflation with negative airway pressure and subsequent airway opening occlusion maintained for 10 to $15 \mathrm{~min}$ up to absorption atelectasis of the whole lung. Lung degassing is intended to help the lavage fluid reach the alveoli more easily and evenly.

\section{Patient position}

The position of the patient during WLL varied considerably among centres: $12(60 \%)$ centres utilized a supine position; 6 (30\%) centres utilized a full lateral position at $90^{\circ} ; 2$ centres utilized a moderate lateral position at $30^{\circ}$ to $45^{\circ}$ inclination. Of those utilizing a lateral position, all but one centre ventilated the non-dependent lung and lavaged the dependent lung. Only one centre uses a combination of full lateral position, dependent lung ventilation and non-dependent lung lavage, having observed better oxygenation due to better ventilation/ perfusion matching, no problems with lavage spillover and more convenient access of the lavaged lung for manual chest percussion. Moreover, 7 centres indicated that patient positioning included the Trendelenburg position to improve lavage recovery and increase alveolar clearance.

\section{Lavage fluid and administration}

All Centres used saline warmed to $37{ }^{\circ} \mathrm{C}$ for WLL. Supplements used at some centres included (number of centres using the supplement): $\mathrm{N}$-acetyl cysteine (1), aminophylline (1), hydrocortisone (1), sodium bicarbonate (2) and gaseous oxygen (1). One centre modified the filling-drainage cycle by adding manual ventilation of the lavaged lung half-way through the draining time, intended to improve the removal of material from the lung [7]. All centres infused saline by gravity. One centre used a specified hydrostatic head of $30 \mathrm{~cm}$ relative to the mid-thorax.

The volume of saline aliquots infused during repeated, sequential lung lavage varied greatly, ranging from 80 (SLBL) to $1,650 \mathrm{ml}$ (WLL), with an average of $800 \pm$ $331 \mathrm{ml}$. The total lavage volume also varied widely among centres, ranging from 5 to 40 litres with most $(13 / 20)$ centres using less than 18 litres per lung (Fig. 3). The mean total volume among the 20 centres was $15.4+/-6.8$ litres per lung. Only one centre regularly utilized a fixed volume (12 litres per lung).

\section{Chest percussion}

Most (14/20) centres used chest percussion to emulsify the PAP sediment ( $90 \%$ lipid) in order to improve therapeutic efficiency. However, the method and timing varied greatly. Ten $(50 \%)$ centres utilized manual chest percussion and four (20\%) centres used mechanical percussion. The timing of chest percussion also varied with 


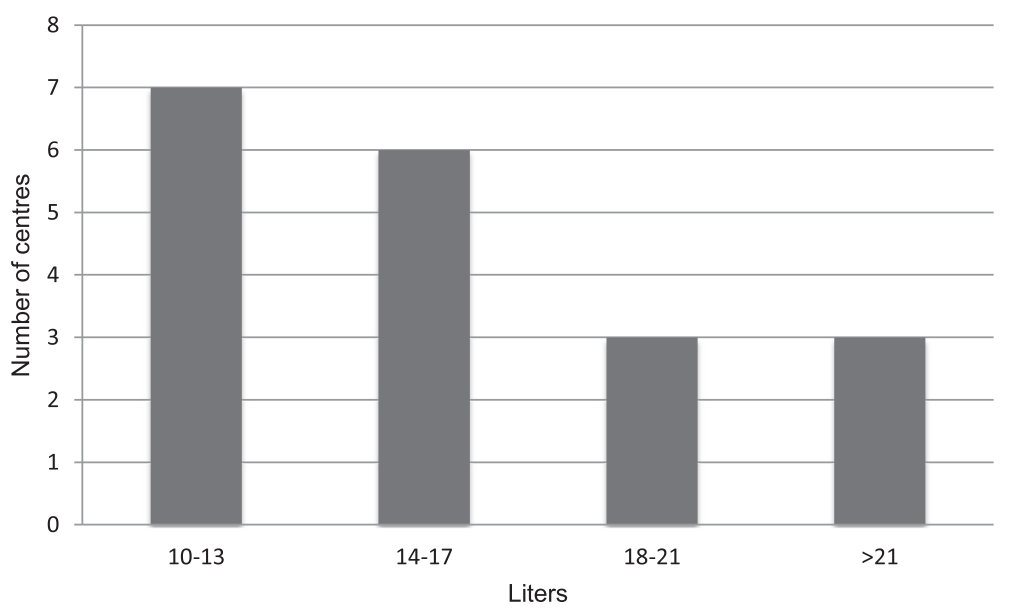

Fig. 3 Stratification of total amount of fluid (litres) used to lavage a single lung

some centres utilizing it during the entirety of each infusion-drainage cycle, some during the drainage portion only, some only after filling.

\section{Duration and termination}

The duration of WLL for one lung varied from 2 to $6 \mathrm{~h}$ with an average of $6+/-1 \mathrm{~h}$ for both lungs. All centres terminated WLL when the drained fluid became markedly less cloudy than observed at the start of therapy; this was usually based on visual inspection of the gross appearance of the recovered fluid. Two centres also used an objective measure of protein content.

\section{Recovery period}

Mechanical ventilation of both lungs in the intensive care unit (ICU) was maintained from $20 \mathrm{~min}$ to $19 \mathrm{~h}$ after completion of WLL, with an average of $5+/-1.5 \mathrm{~h}$. Most patients were discharged from the ICU within $5 \mathrm{~h}$ after extubation.

\section{Lung Lavage in Paediatric Patients}

Seven centres reported performing lung lavage in paediatric patients (4 - WLL or SLBL, 3 only WLL). Three additional centres perform lung lavage only by bronchoscopy (Table 1). The mean (+/-SEM) duration of experience was $16+/-17$ years, but this varied among centres (not shown). The mean number of SLBL procedures performed annually per centre was 5.75 +/- 5.83 and also varied among centres (not shown). The average number of WLL treatments per paediatric PAP patient $(2.3 \pm 1.52)$ was similar to that of adults $(2.5 \pm 1.48)$. Indicators for pediatric WLL were also similar to those for adults. The interval between WLL treatment for each lung was on $5+/-2.8$ days. All centres used warm saline without additives. The volume of saline infused ranged from 250 to $500 \mathrm{ml}$ per aliquot. One centre infused saline under a hydrostatic pressure of $30-40 \mathrm{~cm}$ $\mathrm{H}_{2} \mathrm{O}$. The total volume applied per lung varied from 4 to 14 litres. Three centres applied chest percussion, two of them manually and one mechanically either during the filling or drainage periods. The average duration of WLL in children was $3.5 \mathrm{~h}$. Smaller patients were weaned from mechanical ventilation over a period of between 2 and $48 \mathrm{~h}$ with a mean of $12.7+/-23.5 \mathrm{~h}$. Follow up evaluation after WLL was similar to that of adult PAP patients except that $\mathrm{CT}$ scans were not routinely used. In contrast to adults, paediatric PAP patients received a greater number of lavage procedures (Fig. 4), possibly because of differing disease (i.e., GM-CSFRa deficiency instead of autoimmune PAP) or the more frequent use of SLBL, which treats only a segment or lobe, rather than WLL, which treats the entire lung. At one centre, the intubation method for children, as well as the decision to perform WLL or lobar/ segmental lavage via bronchoscopy, is based on patient size. In children large enough to possibly tolerate a double lumen tube, single-lumen endotracheal tubes of increasing diameter are first 'test-fitted' to identify the maximum possible size while avoiding subglottic trauma. Intubation is then performed with a double-lumen tube of the size identified. For children too small for a double-lumen tube, intubation of the lung to be lavaged is achieved with a single-lumen endotracheal tube, while ventilation of the other lung is achieved by ventilating the trachea around the endotracheal tube. The most frequent complications of WLL in children are reported in Table 5.

\section{Discussion}

As a first step in developing an evidence-based, bestpractice approach to standardizing WLL therapy in PAP, we made the results of a global survey of physicians performing WLL in adults and children. Respondents 


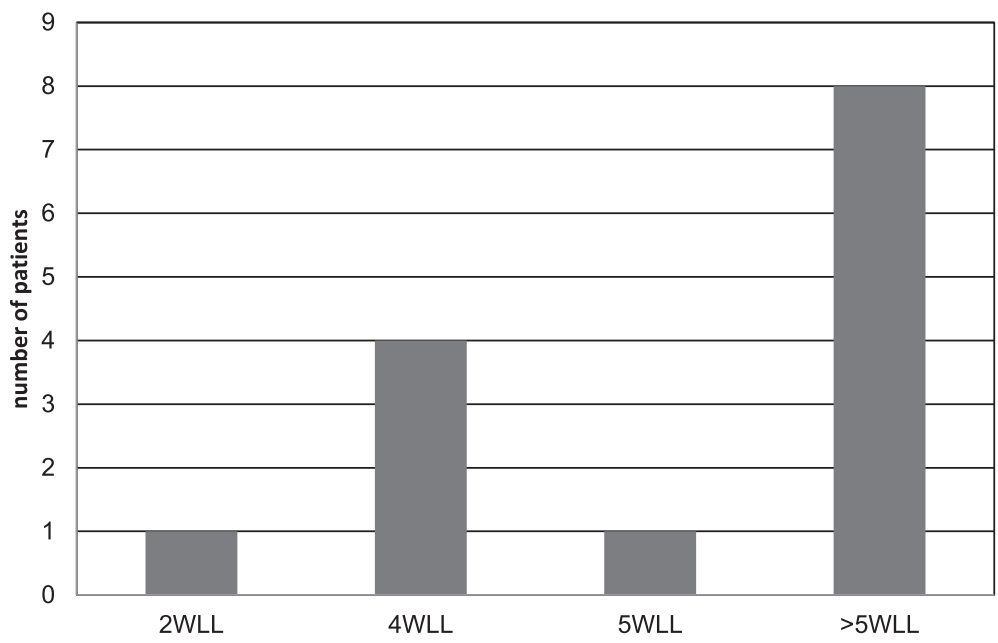

Fig. 4 Stratification of paediatric PAP patients according to the number of procedures received

included 20 centres in 14 countries performing WLL in adults and 10 centres in 6 countries performing WLL in paediatric patients. Some aspects of WLL were similar among centres, including the method for selecting the first lung to be treated, treatment of each lung at different sessions separated by several days to weeks, use of general anesthesia, a double-lumen endotracheal tube (in adults), saline warmed to $37^{\circ} \mathrm{C}$, and drainage of lung lavage fluid by gravity. Other aspects of the procedure varied significantly among centres, including indications for treatment, contraindications, methods and timing of the follow up evaluation, patient position during WLL, the volume of saline infused, use of chest percussion, timing of extubation after WLL and lung isolation and lavage methods for small children. The length of experience among centres varied widely, but the overall rate of serious, procedure-related complications was low. Limited number of diseases requiring WLL therapy, procedural variations and their relationship to differences in outcomes among patients and centres, all support the need for integrated data from multiple centres in order to develop a best-practices approach to standardizing WLL therapy.

Table 5 Complications of WLL in children

\begin{tabular}{ll}
\hline Complication & Overall occurrence rate \\
\hline Hypoxemia & $13 \%$ \\
Fluid leakage & $6 \%$ \\
Pleural effusion & $6 \%$ \\
Fever & $5 \%$ \\
Wheezing & $3 \%$ \\
Pneumonia & $3 \%$ \\
Pneumothorax & $1 \%$ \\
\hline
\end{tabular}

Important findings of our WLL survey include: in spite of its clear therapeutic usefulness, it is not available at most medical centres; it is frequently taught by informal apprenticeship or is even self-taught and it varies among centres with respect to the procedure itself, indications for use, and assessment of benefit. We found 161 reports on the use of WLL therapy for PAP in the NCBI PubMed database including several technical descriptions [8-12]. There was a consensus on indications for WLL therapy in PAP, which included worsening of lung function/gas exchange (100 \% of centres), followed by radiographic evidence of deterioration (79\%), and then symptomatic worsening ( $42 \%$ of centres). Notwithstanding this consensus, the parameter used to determine each varied considerably among centres. Even though the majority of centres used ca. $800 \mathrm{ml}$ of warmed saline for each single infusion during WLL, centres varied in their use and choice of additives, and the total volume of saline used to treat each lung. These differences have important implications for the conduct of clinical trials evaluating new therapeutic approaches.

Interestingly, only $65 \%$ of the centres employed an observation period before performing the WLL. With the exception of rapidly progressive cases, this would be advisable as spontaneous improvement is possible. Even though, complete spontaneous remission, previously believed to occur in up to $30 \%$ of cases [13], is now known not to exceed $10 \%$ of cases [14, 15].

The absence of an association between variability in the clinical course among PAP patients (indicated by the different number of WLL treatments needed to obtain remission) and differences WLL practice among centres (volume of saline used, number of procedures performed annually, length of experience performing WLL) suggest that intrinsic disease characteristics predominate over 
technical differences and experience in applying WLL among centres. On the other hand, the low number of complications and absence of a correlation with experience status may be related to the fact that the centres filling out the questionnaire were all relatively experienced. In fact, several authors stated that they treated patients who were sent for a tertiary referral after a complicated WLL procedure in an inexperienced hospital.

Although WLL is an invasive procedure, it has been determined to be safe and associated with a low rate of procedure-related morbidity ( $18 \%$, including, in order of decreasing frequency, fever, spillage of saline into the ventilated lung, and worsened hypoxemia). SLBL [16] is an alternative to WLL and is regarded as less invasive, but neither its therapeutic effectiveness, nor its rate of complications have been adequately studied and thus was not considered in the present study.

This study has some limitations, which interfere with the statistical analysis of the results, notably: the incomplete participation of physicians/centres who are known to practice WLL therapy. Thus, our results may not adequately reflect the practice of WLL at all centres or, potentially, important improvements made at centres not surveyed. Notwithstanding these limitations, these results provide the first useful data for developing consensus documents regarding technical implementation, indications, and evaluation of WLL therapy. For this reason, the questionnaire has been included in an Additional file 1 for use by other centres wishing to provide additional data regarding their centre's practice of WLL. Even though few centres performing WLL in children were identified/included, one important problem identified with this survey was the lack of availability of double lumen endotracheal tubes of sufficiently small size for use in small children.

\section{Conclusions}

Our study (as well as the literature) provides data for the development of a consensus document and experiencebased, best-practice standardization of WLL. We conclude that the assembly of an international task force on the conduct of WLL therapy in adults and children would be helpful in this regard.

\section{Additional files}

Additional file 1: Therapeutic lavage for pulmonary alveolar proteinosis A questionnaire for an international survey. (DOCX $126 \mathrm{~kb}$ )

Additional file 2: Pharmacologic approaches utilized to support WLL. (DOCX $14 \mathrm{~kb}$ )

\section{Abbreviations}

CEA, carcinoembryonic antigen; CFA, cryptogenic fibrosing alveolitis; CT, computed tomogram; Cyfra 21-1, cytokeratin 19 fragment; DLCO, carbon monoxide diffusing capacity; FVC, forced vital capacity; GM-CSF, granulocytemacrophage colony-stimulating factor; ICU, intensive care unit; IQR, interquartile range; $\mathrm{KL}-6$, Krebs von den Lungen 6; LDH, lactate dehydrogenase; LSBL, lobar or segmental bronchoscopic lavage; NCBI, National Center for Biotechnology Information; PAP, pulmonary alveolar proteinosis; SPA, surfactant protein A; SPD, surfactant protein $\mathrm{D} ; \mathrm{SpO}_{2}$, resting oxygen saturation; WLL, whole lung lavage

\section{Acknowledgements}

This project was started by the late Dr. Maurizio Luisetti (1953 to 2014) whose passion for research and advocacy for people affected by rare diseases, including PAP, and recognition of the need and importance of optimizing and standardizing the practice of $W L L$, resulted in this project. The manuscript was completed by his collaborators and friends who want to honour his memory. We thank all of the physicians at centres performing WLL for participating in the survey.

Members of the Whole lavage International Study Group

Arai Toru, Department of Diffuse Lung Diseases and Respiratory Failure,

Clinical Research Centre, National Hospital Organization Kinki-Chuo Chest Medical Centre, Osaka, Japan

Bazhanov Andrey A., Pulmonology Department of Pavlov State Medical University, St. Petersburg, Russian Federation

Ben-Dov Issahar, Pulmonary Institue' Sheba Medicak Centre, Tel-Hashomer, Tel Aviv University, Sackler Med School, Israel

Bonella Francesco, Department of Pneumology/Allergy, Ruhrlandklinik-

University of Duisburg Essen, Germany

Braschi Antonio, Department of Anaesthesiology and Intensive Care Unit, Fondazione IRCCS Policlinico San Matteo, University of Pavia, Pavia, Italy Casey Alicia, Children's Hospital Boston, Division of Respiratory Diseases, Boston, MA, USA

Costabel Ulrich, Department of Pneumology/Allergy, Ruhrlandklinik-University of Duisburg Essen, Germany

Cottin Vincent, National Reference centre for rare pulmonary disease, Hopital Louis Pradel, Lyon, France

Dogru Deniz, Hacettepe University, Faculty of Medicine, Paediatric Pulmonary Medicine Unit, Sihhiye, Ankara, Turkey

Gesierich Wolfgang, Facharzt für Innere Medizin/Pneumologie, Oberarzt der Klinik für Pneumologie, Leiter der Endoskopie, Asklepios-Fachkliniken

München Gauting, Germany

Griese Matthias, Kinderklinik und Kinderpoliklinik im Dr. von Haunerschen Kinderspital, University of Munich, Germany

Grutters Jan C., Centre of Interstitial Lung Diseases (CIL), St. Antonius Ziekenhuis Nieuwegein, The Netherlands

Halme Maija, Helsinki University Central Hospital, Division of Respiratory Medicine, Department of Medicine, Helsinki, Finland

Henry Michael, Department of Respiratory Medicine, Cork University Hospital Wilton, Cork, Ireland

Herth Felix JF, Pneumology and Respiratory Care Medicine Thoraxklinik, University of Heidelberg, Germany

Hui-ying Wang, Division of Allergy, Department of Internal Medicine, the Second Affiliated Hospital of Zhejiang University Medical College, Hangzhou, Zhejiang, P. R. China

Ichiwata Toshio, Tokyo Medical University Hachioji Medical Centre, Department of Respiratory Medicine, Tokyo, Japan

Ilkovich Julia M., Pulmonology Department of Pavlov State Medical University, St. Petersburg, Russian Federation

Inoue Yoshikazu, Department of Diffuse Lung Diseases and Respiratory Failure, Clinical Research Centre, National Hospital Organization Kinki-Chuo Chest Medical Centre, Osaka, Japan

lotti Giorgio A., Department of Anaesthesiology and Intensive Care, Fondazione IRCCS Policlinico San Matteo, Pavia, Italy

Irani Sarosh, Pulmonary Department, Kantonsspital Aarau, Switzerland Kolek Vítězslav, Dept. of Respiratory Medicine, University Hospital, Olomouc, Czech Republic

Morais António, Serviço de Pneumologia- Hospital São João-Porto, Portugal Morgan Cliff, Department of Critical Care \& Anaesthesia, Royal Brompton Hospital, London, UK

Nicolai Thomas, Kinderklinik und Kinderpoliklinik im Dr. von Haunerschen Kinderspital, University of Munich, Germany

Novikova Lubov N., Pulmonology Department of Pavlov State Medical University, St. Petersburg, Russian Federation

Primhak Robert, Sheffield Children's Hospital, Western Bank, Sheffield, UK Reiter Karl, Kinderklinik und Kinderpoliklinik im Dr. von Haunerschen Kinderspital, University of Munich, Germany 
Retsch-Bogart George, Division of Pulmonology, University of North Carolina at Chapel Hill, NC, USA

Rodi Giuseppe, Department of Anaesthesiology and Intensive Care, Fondazione IRCCS Policlinico San Matteo, Pavia, Italy

Russi Eric, Pulmonary Division University Hospital, Zurich, Switzerland Schmitz Jochen, Lungen Clinic Grosshansdorf, Germany

Schön Carola, Kinderklinik und Kinderpoliklinik im Dr. von Haunerschen Kinderspital, University of Munich, Germany

Schumann Christian, Pulmonology,Thoracic Oncology,Sleep and Respiratory Intensive Care Medicine Kempten-Oberallgäu Hospital Immenstadt, Germany Veltkamp Marcel, Centre of Interstitial Lung Diseases (CIL), St. Antonius Ziekenhuis Nieuwegein, The Netherlands

Verwey Charl, Division of Paediatric Pulmonology, Department of Paediatrics, Chris Hani Baragwanath Academic Hospital University of the Witwatersrand, Johannesburg, Republic of South Africa

Wood Robert E., Division of Pulmonary Medicine Cincinnati Children's Hospital, Cincinnati, OH, USA

\section{Funding}

This study was supported by a grant from the European Union E-Rare JTC 2009: EuPAPnet.

\section{Availability of data and material}

The questionnaire used to perform this survey is available in the Additional file 1.

\section{Authors' contributions}

IC, ML, GB an BCT had full access to all of the study data. CT takes responsibility for the integrity of the data and the accuracy of the data analysis. MG, BCT, FB, UC, GR, CHMVM and JG substantially contributed to the study design. $I C, M L, G R$ and $B C T$ drafted and supervised the manuscript. $M G, F B, J G, K N, C H M V M, U C, V C, T I, Y I, A B, G A l$ critically revised the manuscript. All authors read and approved the final manuscript.

\section{Competing interests}

The authors declare that they have no competing interests.

\section{Consent for publication}

Not applicable

\section{Ethics approval and consent to participate}

This survey was born as part of the project entitled EuPAPNet, funded by eRARE Research Programmes on Rare Diseases. We have the approval by our ethics committee regarding the whole EuPAPNet project. The international survey does not collect individual patient data, but averages data from all the participating centres.

\section{Author details}

'Pneumology Unit, Fondazione IRCCS Policlinico San Matteo and University of Pavia, Pavia, Italy. ${ }^{2}$ Kinderklinik und Kinderpoliklinik im Dr. von Haunerschen Kinderspital, University of Munich, Munich, Germany. ${ }^{3}$ Translational Pulmonary Science Centre, Cincinnati Children's Hospital, Cincinnati, OH, USA. ${ }^{4}$ Interstitial and Rare Lung Disease Unit, Ruhrlandklinik University Hospital, University of Duisburg-Essen, Essen, Germany. ${ }^{5}$ Centre of Interstitial Lung Diseases, St. Antonius Hospital Nieuwegein, Nieuwegein, The Netherlands. ${ }^{6}$ Niigata University Medical and Dental School, Niigata, Japan. ${ }^{7}$ National Reference Centre for Rare Pulmonary Disease, Hopital Louis Pradel, Lyon, France. ${ }^{8}$ Tokyo Medical University Hachioji Medical Center, Tokyo, Japan. ${ }^{9}$ Department of Diffuse Lung Diseases and Respiratory Failure, Clinical Research Centre, National Hospital Organization Kinki-Chuo Chest Medical Centre, Osaka, Japan. ${ }^{10}$ Department of Anesthesiology and Intensive Care, Fondazione IRCCS Policlinico San Matteo, University of Pavia, Pavia, Italy. ${ }^{11}$ Clinical Epidemiology and Biometric Unit, Fondazione IRCCS Policlinico San Matteo, Pavia, Italy. ${ }^{12}$ Laboratorio di Biochimica e Genetica, S.C. Pneumologia, Fondazione IRCCS Policlinico San Matteo, via Taramelli 5, 27100 Pavia, Italy.

Received: 23 April 2016 Accepted: 3 August 2016

Published online: 31 August 2016

\section{References}

1. Ramirez J, Schultz RB, Dutton RE. Pulmonary Alveolar Proteinosis: a new technique and rationale for treatment. Arch Intern Med. 1963;112:419-31.

2. Rosen SG, Castelman B, Liebow AA. Pulmonary alveolar proteinosis. N Engl J Med. 1958;258:1123-42.

3. Trapnell BC, Whitsett JA, Nakata K. Pulmonary alveolar proteinosis. N Engl J Med. 2003;349:2527-39.

4. Seymour JF, Presneill JJ. Pulmonary alveolar proteinosis: progress in the first 44 years. Am J Respir Crit Care Med. 2002;166:215-35.

5. Luisetti M, Kadija Z, Mariani F, et al. Therapy options in pulmonary alveolar proteinosis. Ther Adv Respir Dis. 2010:4:239-48.

6. Luisetti M. Call for an International survey on therapeutic lavage for pulmonary alveolar proteinosis. Eur Respir J. 2012;39:1049.

7. Bingisser R, Kaplan V, Zollinger A, et al. Whole-lung lavage in alveolar proteinosis by a modified lavage technique. Chest. 1998;113:1718-9.

8. Ben-Abraham R, Greenfeld A, Rozenman J, et al. Pulmonary alveolar proteinosis: step-by-step perioperative care of whole lung lavage procedure. Heart Lung. 2002;31:43-9.

9. Michaud G, Reddy C, Ernst A. Whole lung lavage for pulmonary alveolar proteinosis. Chest. 2009;136:1678-81.

10. Silva A, Moreto A, Pinho C, et al. Bilateral whole lung lavage in pulmonary alveolar proteinosis. A retrospective study. Rev Port Pneumol. 2014;20:254-9.

11. Bonella F, Bauer PC, Griese M, et al. Wash-out kinetics and efficacy of a modified lavage technique for alveolar proteinosis. Eur Respir J. 2012;40:1468-74.

12. Rodi G, lotti G, Galbusera C, et al. Whole lung lavage. Monaldi Arch Chest Dis. 1995;50:64-6.

13. Prakash UB, Barham SS, Carpenter HA, et al. Pulmonary alveolar phospholipoproteinosis: experience with 34 cases and a review. Mayo Clin Proc. 1987;62:499-518.

14. Bonella F, Bauer PC, Griese M, et al. Pulmonary alveolar proteinosis: new insights from a single-center cohort of 70 patients. Respir Med. 2011;105:1908-16.

15. Campo I, Mariani F, Rodi G, et al. Assessment and management of pulmonary alveolar proteinosis in a reference center. Orphanet J Rare Dis. 2013;8:40-6

16. Cheng SL, Chang HT, Lau HP, et al. Pulmonary alveolar proteinosis: treatment by bronchofiberscopic lobar lavage. Chest. 2002;122:1480-5.

\section{Submit your next manuscript to BioMed Central and we will help you at every step:}

- We accept pre-submission inquiries

- Our selector tool helps you to find the most relevant journal

- We provide round the clock customer support

- Convenient online submission

- Thorough peer review

- Inclusion in PubMed and all major indexing services

- Maximum visibility for your research

Submit your manuscript at www.biomedcentral.com/submit
Ciomed Central 